\title{
Toy model for two chiral nonets
}

SU-4252-810

\author{
Amir H. Fariborz $\mathbf{a} \ddagger$, Renata Jora $\mathbf{b} \dagger$, and Joseph Schechter $\mathbf{b} \S$ \\ ${ }^{a}$ Department of Mathematics/Science, State University of New York Institute of Technology, Utica, NY 13504-3050, USA \\ ${ }^{b}$ Department of Physics, Syracuse University, Syracuse, NY 13244-1130, USA
}

(Dated: August 13, 2018)

\begin{abstract}
Motivated by the possibility that nonets of scalar mesons might be described as mixtures of "two quark" and "four quark" components, we further study a toy model in which corresponding chiral nonets (containing also the pseudoscalar partners) interact with each other. Although the "two quark" and "four quark" chiral fields transform identically under $\mathrm{SU}(3)_{L} \times \mathrm{SU}(3)_{R}$ transformations they transform differently under the $\mathrm{U}(1)_{A}$ transformation which essentially counts total (quark + antiquark) content of the mesons. To implement this we formulate an effective Lagrangian which mocks up the $\mathrm{U}(1)_{A}$ behavior of the underlying QCD. We derive generating equations which yield Ward identity type relations based only on the assumed symmetry structure. This is applied to the mass spectrum of the low lying pseudoscalars and scalars. as well as their "excitations". Assuming isotopic spin invariance, it is possible to disentangle the amount of "two quark" vs. "four quark" content in the pseudoscalar $\pi, K, \eta$ type states and in the scalar $\kappa$ type states. It is found that a small "four quark" content in the lightest pseudoscalars is consistent with a large "four quark" content in the lightest of the scalar $\kappa$ mesons. The present toy model also allows one to easily estimate the strength of a "four quark" vacuum condensate. There seems to be a rich and interesting structure.

PACS numbers: 13.75.Lb, 11.15.Pg, 11.80.Et, 12.39.Fe
\end{abstract}

\section{INTRODUCTION}

The last few years have seen a renewal of interest [1]- [30] in the low energy scalar sector of QCD. Many physicists now believe in the existence of the light, broad $I=J=0$ resonance, sigma in the $500-600 \mathrm{MeV}$ region as well as a light broad $I, J=1 / 2,0$ resonance, kappa in the $700-900 \mathrm{MeV}$ region. Together with the well established $f_{0}(980)$ and $a_{0}(980)$ scalar resonances, these comprise a putative nonet of "elementary particles". Furthermore, this nonet seems likely to have a quark structure like $q q \bar{q} \bar{q}$ rather than the conventional $q \bar{q}[31$. This of course raises the question of where are the conventional $q \bar{q}$ p-wave scalars expected in the quark model. Arguments have been given [32] that the experimental data are better fit when the two scalar nonets mix with each other and the resulting "level repulsion", pushes the conventional scalars to higher masses than otherwise expected.

In order to further explore the feature of mixing between $q \bar{q}$ type and $q q \bar{q} \bar{q}$ type states it seems interesting to consider a linear $\mathrm{SU}(3) \times \mathrm{SU}(3)$ sigma model which contains also the pseudoscalar nonet partners of these two scalar nonets. Parenthetically, we remark that while the non- linear sigma model [33, 34] and its extension to the chiral perturbation theory program [35] are often more efficient for systematic calculations, linear sigma models have a very long history of furnishing important insights into the nature of strong hadron dynamics. The $\mathrm{SU}(2)$ linear sigma model was first given in ref. 33|. It was used as a basis for understanding the current algebra treatment of $\pi \pi$ scattering near threshold in ref. [36]. The SU(3) version was given in the first of ref. [37]. A detailed application to the low energy pseudoscalar mass spectrum was given [38] before QCD in which, among other things, it was shown how a $\mathrm{U}(1)_{A}$ violating term natural in the $\mathrm{SU}(3)$ model could solve the $\eta^{\prime}$ problem. Such a term was later discovered to arise from instanton effects [39]. The connection was pointed out in ref. [40] and emphasized by 't Hooft [41].

The model containing two different chiral nonets to be discussed here was proposed in section V of ref. 29] and an initial treatment, neglecting flavor symmetry breaking, was given. A discussion, taking the flavor symmetry breaking into account has very recently been presented in ref. 42]. Actually, it turns out that the model is very complicated since many different terms can be included and various assumptions about the nature of the symmetry breaking can be made. In this paper we will set up the formalism for treating consequences of the model which hold (at tree level) just due to the symmetry structure of the model and will give a numerical treatment using what might be the simplest choice of symmetry breaking terms.

Section II begins with a review of the flavor transformation properties of the two chiral nonet fields, $M$ and $M^{\prime}$

\footnotetext{
¥ Email: fariboa@sunyit.edu

$\dagger$ Email: cjora@physics.syr.edu

$\S$ Email: schechte@physics.syr.edu
} 
which are used in the model. Each contains nine pseudoscalar and nine scalar fields. Under chiral $\mathrm{SU}(3)_{L} \times \mathrm{SU}(3)_{R}$ transformations both fields transform in an identical manner. Thus a chiral Lagrangian which respects only this symmetry cannot directly distinguish between a "two quark" (i.e. $q \bar{q}$ ) or a "four quark" scalar, for example. However, it is noted again that the $\mathrm{U}(1)_{A}$ transformation actually counts the number of quarks in these mesons and provides a way to distinguish them. In order to make use of this, the Lagrangian should of course be set up appropriately. We implement this by requiring that the Lagrangian mock up the anomalous $\mathrm{U}(1)_{A}$ equation of the underlying $\mathrm{QCD}$ and that the analogs of the quark mass terms also mock up the $\mathrm{U}(1)_{A}$ transformation properties of the quark mass terms in the underlying theory. A reasonable initial thought on which terms to include in the Lagrangian is to restrict it to be renormalizable. It is noted, with details in Appendix A, that the renormalizable $M-M^{\prime}$ Lagrangian has however very many more terms than does the renormalizable single $M$ Lagrangian. An alternate way, which still satisfies generality, is to consider any number of terms, renormalizable or not, and just use the information which follows from the symmetry behavior of the Lagrangian.

In order to exploit this symmetry information we derive, in section III, vector type and axial vector type "generating equations" for the model. These can be differentiated with respect to the fields to yield many tree level Ward identities which are independent of the number of symmetric terms included in the Lagrangian. In addition to the analog of "two quark" condensates which occur in the single $M$ model, the present model also brings "four quark" condensates into the picture.

In section IV, we derive predictions for the mass spectrum which follow from this symmetry approach. The characteristic feature is mixing between "two quark" and "four quark" mesons with the same quantum numbers. Assuming isospin invariance, predictions are made for the $\pi-\pi^{\prime}$ mixing sector, the $K-K^{\prime}$ mixing sector, the strange scalar $\kappa-\kappa^{\prime}$ mixing sector and the sector involving mixing of the four isocalar pseudoscalars ( $\eta$ type particles). It is shown how to formulate the first three of these mixing sectors in a parallel and economical way.

In section $\mathrm{V}$, the mass spectrum relations are compared with experiment. First the three $2 \times 2$ mixing sectors are treated. The inputs are taken to be the six masses of the well known and not so well known particles, the pion and kaon decay constants and a model parameter denoted $x_{\pi}$, which is the squared mass of the unmixed (or "bare") pion. These are enough to determine all the relevant parameters of these three systems. The pseudoscalar mixing is very sensitively dependent on $x_{\pi}$; as it increases from the experimental value, $m_{\pi}^{2}$ the four quark components of the pion and the kaon increase. On the other hand, the scalar $\kappa$ has a large four quark component. This feature thus provides some support for a more exotic structure of the low lying scalars. Another interesting feature of the present model, discussed in this section, is that it permits one to estimate the strength of a four quark vacuum condensate. Finally, section V contains a brief summary, the connection with other results on the same model and directions for future work.

\section{SYMMETRIES AND LAGRANGIAN}

First, let us briefly review 29] the fields of the model and their transformation properties. The schematic structure for the matrix $M(x)$ realizing a $q \bar{q}$ composite in terms of quark fields $q_{a A}(x)$ can be written

$$
M_{a}^{b}=\left(q_{b A}\right)^{\dagger} \gamma_{4} \frac{1+\gamma_{5}}{2} q_{a A},
$$

where $a$ and $A$ are respectively flavor and color indices. Our convention for matrix notation is $M_{a}^{b} \rightarrow M_{a b}$. Then $M$ transforms under chiral $\mathrm{SU}(3)_{L} \times \mathrm{SU}(3)_{R}$ as

$$
M \rightarrow U_{L} M U_{R}^{\dagger}
$$

where $U_{L}$ and $U_{R}$ are unitary, unimodular matrices associated with the transformations on the left handed $\left(q_{L}=\frac{1}{2}\left(1+\gamma_{5}\right) q\right)$ and right handed $\left(q_{R}=\frac{1}{2}\left(1-\gamma_{5}\right) q\right)$ quark projections. For the discrete transformations charge congugation $C$ and parity $P$ one verifies

$$
C: \quad M \rightarrow M^{T}, \quad P: \quad M(\mathbf{x}) \rightarrow M^{\dagger}(-\mathbf{x}) .
$$

The $\mathrm{U}(1)_{A}$ transformation acts as $q_{a L} \rightarrow e^{i \nu} q_{a L}, q_{a R} \rightarrow e^{-i \nu} q_{a R}$ and results in:

$$
M \rightarrow e^{2 i \nu} M \text {. }
$$

Next, consider the $q q \bar{q} \bar{q}$ type fields. One interesting model [43] postulates that the light scalars are "molecules" made out of two pseudoscalar mesons. The chiral realization of this picture would result in the following schematic structure:

$$
M_{a}^{(2) b}=\epsilon_{a c d} \epsilon^{b e f}\left(M^{\dagger}\right)_{e}^{c}\left(M^{\dagger}\right)_{f}^{d}
$$


One can verify that $M^{(2)}$ transforms exactly in the same way as $M$ under $\mathrm{SU}(3)_{L} \times \mathrm{SU}(3)_{R}, C$ and $P$. Under $\mathrm{U}(1)_{A}$ it transforms as

$$
M^{(2)} \rightarrow e^{-4 i \nu} M^{(2)},
$$

which differs from Eq. (44). Another interesting approach 31] to explaining the light scalar mesons was formulated by Jaffe in the framework of the MIT bag model. It was observed that the spin-spin (hyperfine) piece of the one gluon exchange interaction between quarks gives an exceptionally strong binding to an s-wave $q q \bar{q} \bar{q}$ scalar state. The scalar states of this type may be formally written as bound states of a "dual quark" and "dual antiquark". There are two possibilities if the dual antiquark is required to belong to a $\overline{3}$ representation of flavor $\mathrm{SU}(3)$. In the first case it belongs to a $\overline{3}$ of color and is a spin singlet. This has the schematic chiral realization,

$$
\begin{aligned}
& L^{g E}=\epsilon^{g a b} \epsilon^{E A B} q_{a A}^{T} C^{-1} \frac{1+\gamma_{5}}{2} q_{b B}, \\
& R^{g E}=\epsilon^{g a b} \epsilon^{E A B} q_{a A}^{T} C^{-1} \frac{1-\gamma_{5}}{2} q_{b B},
\end{aligned}
$$

where $C$ is the charge conjugation matrix of the Dirac theory. A suitable form for the $M$ matrix is:

$$
M_{g}^{(3) f}=\left(L^{g A}\right)^{\dagger} R^{f A} .
$$

$M^{(3)}$ can be seen to transform in the same way as $M^{(2)}$ under $\mathrm{SU}(3)_{L} \times \mathrm{SU}(3)_{R}, C, P$ and $\mathrm{U}(1)_{A}$. In the second case the dual antiquark belongs to a 6 representation of color and has spin 1 . It has the corresponding schematic chiral realization:

$$
\begin{aligned}
& L_{\mu \nu, A B}^{g}=L_{\mu \nu, B A}^{g}=\epsilon^{g a b} q_{a A}^{T} C^{-1} \sigma_{\mu \nu} \frac{1+\gamma_{5}}{2} q_{b B}, \\
& R_{\mu \nu, A B}^{g}=R_{\mu \nu, B A}^{g}=\epsilon^{g a b} q_{a A}^{T} C^{-1} \sigma_{\mu \nu} \frac{1-\gamma_{5}}{2} q_{b B},
\end{aligned}
$$

where $\sigma_{\mu \nu}=\frac{1}{2 i}\left[\gamma_{\mu}, \gamma_{\nu}\right]$. This choice leads to an $M$ matrix

$$
M_{g}^{(4) f}=\left(L_{\mu \nu, A B}^{g}\right)^{\dagger} R_{\mu \nu, A B}^{f},
$$

where the dagger operation includes a factor $(-1)^{\delta_{\mu 4}+\delta_{\nu 4}} \cdot M^{(4)}$ also transforms like $M^{(2)}$ and $M^{(3)}$ under all of $\mathrm{SU}(3)_{L} \times \mathrm{SU}(3)_{R}, C, P$ and $\mathrm{U}(1)_{A}$. The specific form favored by the MIT bag model calculation actually corresponds to a particular linear combination of $M^{(3)}$ and $M^{(4)}$. Furthermore one can verify that $M^{(2)}$ in Eq. (5) is related by a Fierz transformation to a linear combination of $M^{(3)}$ and $M^{(4)}$. Thus only two of $M^{(2)}, M^{(3)}$ and $M^{(4)}$ are linearly independent. In any event, at the present effective Lagrangian level, there are no quantum numbers to distinguish $M^{(2)}, M^{(3)}$, and $M^{(4)}$ from each other so we may as well just denote an arbitrary linear combination of them to be our $q q \bar{q} \bar{q}$ field, $M^{\prime}$. Note that $M$ and $M^{\prime}$ are distinguished from each other by their different $\mathrm{U}(1)_{A}$ transformation properties. These fields may be decomposed into hermitian scalar (S) and pseudoscalar $(\phi)$ nonets as,

$$
\begin{aligned}
M & =S+i \phi, \\
M^{\prime} & =S^{\prime}+i \phi^{\prime} .
\end{aligned}
$$

We will be interested in the situation where non-zero vacuum values of the diagonal components of $S$ and $S^{\prime}$ may exist. These will be denoted by,

$$
\left\langle S_{a}^{b}\right\rangle=\alpha_{a} \delta_{a}^{b}, \quad\left\langle S_{a}^{\prime b}\right\rangle=\beta_{a} \delta_{a}^{b}
$$

In the iso-spin invariant limit, $\alpha_{1}=\alpha_{2}$ and $\beta_{1}=\beta_{2}$ while in the $\mathrm{SU}(3)$ invariant limit, $\alpha_{1}=\alpha_{2}=\alpha_{3}$ and $\beta_{1}=\beta_{2}=\beta_{3}$.

The Lagrangian density which defines our model is

$$
\mathcal{L}=-\frac{1}{2} \operatorname{Tr}\left(\partial_{\mu} M \partial_{\mu} M^{\dagger}\right)-\frac{1}{2} \operatorname{Tr}\left(\partial_{\mu} M^{\prime} \partial_{\mu} M^{\prime \dagger}\right)-V_{0}\left(M, M^{\prime}\right)-V_{S B}
$$

where $V_{0}\left(M, M^{\prime}\right)$ stands for a general function made from $\mathrm{SU}(3)_{L} \times \mathrm{SU}(3)_{R}$ (but not necessarily $\left.\mathrm{U}(1)_{A}\right)$ invariants formed out of $M$ and $M^{\prime}$. Furthermore $V_{S B}$ is taken to be a flavor symmetry breaking term which should mock up the quark mass terms which perform this function in the fundamental QCD Lagrangian. Other physical particles (including glueballs) could be added for more realism, but Eq. (13) is already quite complicated. 
To get an initial indication of what is happening in this kind of model the drastically simplified case where the quark mass effective term, $V_{S B}$ is absent and where $V_{0}$ is simply given by:

$$
V_{0}=-c_{2} \operatorname{Tr}\left(M M^{\dagger}\right)+c_{4} \operatorname{Tr}\left(M M^{\dagger} M M^{\dagger}\right)+d_{2} \operatorname{Tr}\left(M^{\prime} M^{\prime \dagger}\right)+e \operatorname{Tr}\left(M M^{\prime \dagger}+M^{\prime} M^{\dagger}\right),
$$

was treated in sec.V of ref. [29]. Here $c_{2}, c_{4}$ and $d_{2}$ are positive real constants. The $M$ matrix field is chosen to have a wrong sign mass term so that there will be spontaneous breakdown of chiral symmetry. A pseudoscalar octet is thus massless. The mixing between the $M$ and $M^{\prime}$ is controlled by the parameter $e$. The first feature found for this simplified model was that the analog, $\left\langle{S^{\prime}}_{a}^{a}\right\rangle$ of the $q q \bar{q} \bar{q}$ condensate in QCD acquired a small non-zero value due to the mixing between $S$ and $S^{\prime}$. The main question is the level ordering. Since the light pseudoscalars (e.g. $\pi^{+}=\phi_{1}^{2}$ ) are naturally identified, before mixing, with the $q \bar{q}$ field $\mathrm{M}$, one wonders whether the two quark rather than the four quark scalars aren't the lightest ones. It was found however that it is natural (but not unique) in the model to have the energy level pattern in ascending order- pseudoscalar Nambu-Goldstone boson with primarily $q \bar{q}$ structure, scalar with primarily $q q \bar{q} \bar{q}$ structure, pseudoscalar with primarily $q q \bar{q} \bar{q}$ structure and scalar with primarily $q \bar{q}$ structure. These refer to degenerate octets which are each mixtures of $M$ and $M^{\prime}$ states. This seems to be similar to the expected experimental pattern and gives us some motivation to proceed further.

The next question is what terms to include in the Lagrangian Eq. (13). A natural first attempt would be to consider a renormalizable model in which $V_{0}$ contains all the $\mathrm{SU}(3) \times \mathrm{SU}(3)$ invariant terms up to four powers of the fields. These are listed in Appendix A. It is seen that there are 21 terms of this type. This is a rather large number and while not impossible to handle suggests trying another tack. We will just allow $V_{0}$ to contain all possible terms which are $\mathrm{SU}(3)_{L} \times \mathrm{SU}(3)_{R}$ symmetric and use the information provided by this symmetry. This is more general and also allows for non-renormalizable terms. The price to be paid is that we only get information which follows just from the symmetry structure. In an earlier treatment [38] of the single chiral nonet case, it was found that the results obtained were essentially those which could be obtained from the "current algebra" approach. Furthermore, we will try to make use of the fact that $M$ and $M^{\prime}$ have different $\mathrm{U}(1)_{A}$ transformation properties. We thus demand that the Lagrangian without $V_{S B}$ mock up the anomalous $\mathrm{U}(1)_{A}$ equation of QCD,

$$
\delta \mathcal{L}=G,
$$

where $\delta$ denotes the axial $\mathrm{U}(1)$ variation and $G$ is proportional to the product of the QCD field strength tensor and its dual. This can be achieved by making all of the terms in $V_{0}$, except for a limited number, $\mathrm{U}(1)_{A}$ invariant. The special terms will be constructed to satisfy Eq. (15). An example of a term which is not $\mathrm{U}(1)_{A}$ invariant is the mixing term used in the simplified model above: $\operatorname{Tr}\left(M^{\prime} M^{\dagger}\right)+$ h.c.. However a mixing term of the type:

$$
\epsilon_{a b c} \epsilon^{\text {def }} M_{d}^{a} M_{e}^{b} M_{f}^{\prime c}+\text { h.c. }
$$

is $\mathrm{U}(1)_{A}$ invariant and hence possibly the most important one.

$\mathrm{An} \mathrm{SU}(3)_{L} \times \mathrm{SU}(3)_{R}$ invariant but not $\mathrm{U}(1)_{A}$ invariant term which mocks up Eq. (15) can be seen 44] to be

$$
\mathcal{L}_{\text {anom }}=\frac{i G}{12} \ln \left(\frac{\operatorname{det} M}{\operatorname{det} M^{\dagger}}\right) .
$$

Here, $G$ is being formally considered as an effective pseudoscalar glueball field in the effective Lagrangian. To get an $\eta^{\prime}(958)$ mass term in the effective lagrangian framework one can [44] include a wrong sign mass term for G: $c G^{2} / 2$ in the Lagrangian which of course does not change the flavor symmetry structure. Then integrating out $G$ yields the effective $\eta(960)$ mass term:

$$
\mathcal{L}_{\eta}=-c_{3}\left[\ln \left(\frac{\operatorname{det} M}{\operatorname{det} M^{\dagger}}\right)\right]^{2},
$$

where $c_{3}=-1 /(288 c)$. The nature of this term becomes more apparent when one goes to the non-linear realization where $M \rightarrow \alpha_{1} \exp \left(i \phi / \alpha_{1}\right)$. For the present paper we shall consider this to be the only $\mathrm{SU}(3)_{L} \times \mathrm{SU}(3)_{R}$ invariant but not $\mathrm{U}(1)_{A}$ invariant term. However, it is not at all unique when we consider a model with two chiral nonets. For example one can also include something like the non- $\mathrm{U}(1)_{A}$ invariant mixing term $\operatorname{Tr}\left(M^{\prime} M^{\dagger}\right)+$ h.c. by writing a candidate Lagrangian piece:

$$
\frac{i G}{12}\left[\gamma_{1} \ln \left(\frac{\operatorname{det} M}{\operatorname{det} M^{\dagger}}\right)+\gamma_{2} \ln \left(\frac{\operatorname{Tr}\left(M M^{\prime \dagger}\right)}{\operatorname{Tr}\left(M^{\prime} M^{\dagger}\right)}\right]\right.
$$

and proceeding as above. In order to properly mock up the anomaly in this case it is necessary [45] that the real numbers $\gamma_{1}$ and $\gamma_{2}$ satisfy

$$
\gamma_{1}+\gamma_{2}=1
$$


The generalization to more than two such terms is evident. It may be noted that the $M-M^{\prime}$ mixing term resulting from Eq. (19) mixes only the pseudoscalar fields and not the scalar ones.

Finally, let us consider the flavor symmetry breaking terms. To get more restrictions, we assume that such a term should mock up both the $\mathrm{SU}(3)_{L} \times \mathrm{SU}(3)_{R}$ and $\mathrm{U}(1)_{A}$ transformation properties of the quark mass terms in the fundamental QCD Lagrangian. It is convenient to introduce a diagonal matrix,

$$
A=\operatorname{diag}\left(A_{1}, A_{2}, A_{3}\right)
$$

which is proportional to the diagonal matrix made from the three light quark masses, $\operatorname{diag}\left(m_{u}, m_{d}, m_{s}\right)($ See 40$]$ for further details). Then, from Eq. (1), we note an obvious choice for a flavor symmetry breaking term,

$$
V_{S B}=-\operatorname{Tr}\left[A\left(M+M^{\dagger}\right)\right]=-2 \operatorname{Tr}(A S),
$$

which transforms like $\left(3,3^{*}\right)+\left(3^{*}, 3\right)$ under $\mathrm{SU}(3)_{L} \times \mathrm{SU}(3)_{R}$. Under the $\mathrm{U}(1)_{A}$ transformation of Eq. (4), it goes to $-e^{2 i \nu} \operatorname{Tr}(A M)+$ h.c.. Note that the similar simple possibility, $-2 \operatorname{Tr}\left(A S^{\prime}\right)$ does not correctly mock up the U(1) $A$ transformation property of the QCD mass term. However Eq. (22) is not at all unique in correctly mocking up the quark mass term. An interesting term which does mock up the quark mass term also involves mixing and has the form,

$$
\epsilon_{a b c} \epsilon^{\operatorname{def}} A_{d}^{a} M_{e}^{b} M_{f}^{c}+\text { h.c. }
$$

This term mixes both scalars and pseudoscalars but with opposite signs.

For what follows, it is convenient to record the behaviors of the fields under infinitesimal transformations. Let us write the infinitesimal vector $(\mathrm{L}+\mathrm{R})$ and axial vector $(\mathrm{L}-\mathrm{R})$ transformations of $\phi$ and $S$ as,

$$
\begin{gathered}
\delta_{V} \phi=\left[E_{V}, \phi\right], \quad \delta_{A} \phi=-i\left[E_{A}, S\right]_{+}, \\
\delta_{V} S=\left[E_{V}, S\right], \quad \delta_{A} S=i\left[E_{A}, \phi\right]_{+} .
\end{gathered}
$$

Here, unitarity demands that the infinitesimal matrices obey,

$$
E_{V}^{\dagger}=-E_{V}, \quad E_{A}^{\dagger}=-E_{A}
$$

If we demand that the transformations be unimodular, so that the $\mathrm{U}(1)_{A}$ transformation is not included (the $\mathrm{U}(1)_{V}$ transformation is trivial for mesons), we should also impose $\operatorname{Tr}\left(E_{A}\right)=0$. However we will not do this so the effects of $\mathrm{U}(1)_{A}$ will also be included. The transformation properties of the $q q \bar{q} \bar{q}$ type fields are:

$$
\begin{gathered}
\delta_{V} \phi^{\prime}=\left[E_{V}, \phi^{\prime}\right], \quad \delta_{A} \phi^{\prime}=-i\left[E_{A}, S^{\prime}\right]_{+}+2 i S^{\prime} \operatorname{Tr}\left(E_{A}\right), \\
\delta_{V} S^{\prime}=\left[E_{V}, S^{\prime}\right], \quad \delta_{A} S^{\prime}=i\left[E_{A}, \phi^{\prime}\right]_{+}-2 i \phi^{\prime} \operatorname{Tr}\left(E_{A}\right) .
\end{gathered}
$$

The extra terms for the axial transformations reflect the different $\mathrm{U}(1)_{A}$ transformation properties of $M$ and $M^{\prime}$.

\section{GENERATING EQUATIONS}

We shall consider, in this paper, tree level predictions for the Lagrangian of Eq.(13) in which the only U(1) $A$ violating term in $V_{0}$ is that of Eq. (18). The only term in $V_{S B}$ will be taken to be the simplest one given in Eq. (22). In this minimal picture, there is no symmetry breaking associated with the $q q \bar{q} \bar{q}$ fields in $M^{\prime}$. The symmetry breaking in the physical states (which contain two quark as well as four quark components) is due to the mixing terms which, as we have already seen in Eq. (16), can be invariant under $\mathrm{SU}(3) \times \mathrm{SU}(3) \times \mathrm{U}(1)_{A}$.

The method of treatment, as used earlier [38] to discuss the model containing only the field $M$, is based on two generating equations which reflect the invariance of $V_{0}$ under vector and axial vector transformations. Differentiating them once, relates two point vertices (masses) with one point vertices. Differentiating them twice relates three point vertices (trilinear couplings) with masses and so on. These are essentially tree level Ward identities.

Under the infinitesimal vector and axial vector transformations we have,

$$
\begin{aligned}
& \delta_{V} V_{0}=\left\{\operatorname{Tr}\left(\frac{\partial V_{0}}{\partial \phi} \delta_{V} \phi+\frac{\partial V_{0}}{\partial S} \delta_{V} S\right)+(\phi, S) \rightarrow\left(\phi^{\prime}, S^{\prime}\right)\right\}=0 \\
& \delta_{A} V_{0}=\left\{\operatorname{Tr}\left(\frac{\partial V_{0}}{\partial \phi} \delta_{A} \phi+\frac{\partial V_{0}}{\partial S} \delta_{A} S\right)+(\phi, S) \rightarrow\left(\phi^{\prime}, S^{\prime}\right)\right\}=-\mathcal{L}_{\eta},
\end{aligned}
$$


wherein the non-zero value of the axial variation equation reflects the presence in $V_{0}$ of the single $U(1)_{A}$ non-invariant term of Eq. (18). Using Eqs. (24) and (26) as well as the arbitrariness of the variations $E_{V}$ and $E_{A}$ yields the matrix generating equations,

$$
\begin{aligned}
& \left\{\left[\phi, \frac{\partial V_{0}}{\partial \phi}\right]+\left[S, \frac{\partial V_{0}}{\partial S}\right]+(\phi, S) \rightarrow\left(\phi^{\prime}, S^{\prime}\right)\right\}=0, \\
& \left\{\left[\phi, \frac{\partial V_{0}}{\partial S}\right]_{+}-\left[S, \frac{\partial V_{0}}{\partial \phi}\right]_{+}+(\phi, S) \rightarrow\left(\phi^{\prime}, S^{\prime}\right)\right\}=1\left[2 \operatorname{Tr}\left(\phi^{\prime} \frac{\partial V_{0}}{\partial S^{\prime}}-S^{\prime} \frac{\partial V_{0}}{\partial \phi^{\prime}}\right)-8 c_{3} i \ln \left(\frac{\operatorname{det} M}{\operatorname{det} M^{\dagger}}\right)\right],
\end{aligned}
$$

where, in addition, the form of Eq. (18) was used. To get constraints on the particle masses we will differentiate these equations once with respect to each of the four matrix fields: $\phi, \phi^{\prime}, S, S^{\prime}$ and evaluate the equations in the ground state. Thus we also need the "minimum" condition,

$$
\left\langle\frac{\partial V_{0}}{\partial S}\right\rangle+\left\langle\frac{\partial V_{S B}}{\partial S}\right\rangle=0, \quad\left\langle\frac{\partial V_{0}}{\partial S^{\prime}}\right\rangle+\left\langle\frac{\partial V_{S B}}{\partial S^{\prime}}\right\rangle=0 .
$$

Using our present choice of Eq. (22) as the only flavor symmetry breaker and Eq. (12), this becomes

$$
\left\langle\frac{\partial V_{0}}{\partial S_{a}^{a}}\right\rangle=2 A_{a}, \quad\left\langle\frac{\partial V_{0}}{\partial S_{a}^{\prime a}}\right\rangle=0 .
$$

Now let us differentiate successively the vector generating equation with respect to $S_{a}^{b}$ and to ${S^{\prime}}_{a}^{b}$. This gives with the help of Eq.(30), the following two relations:

$$
\begin{aligned}
\left(\alpha_{a}-\alpha_{b}\right)\left\langle\frac{\partial^{2} V_{0}}{\partial S_{b}^{a} \partial S_{a}^{b}}\right\rangle+\left(\beta_{a}-\beta_{b}\right)\left\langle\frac{\partial^{2} V_{0}}{\partial{S^{\prime}}_{b}^{a} \partial S_{a}^{b}}\right\rangle & =2\left(A_{a}-A_{b}\right), \\
\left(\alpha_{a}-\alpha_{b}\right)\left\langle\frac{\partial^{2} V_{0}}{\partial S_{b}^{a} \partial{S^{\prime}}_{a}^{b}}\right\rangle+\left(\beta_{a}-\beta_{b}\right)\left\langle\frac{\partial^{2} V_{0}}{\partial{S^{\prime}}_{b}^{a} \partial{S^{\prime}}_{a}^{\prime}}\right\rangle & =0 .
\end{aligned}
$$

The first of these equations relates the mass mixing transition with the unprimed scalar squared masses while the second of these relates the mass mixing transition with the primed scalar squared masses. It may be seen that information is obtained only for particles with different upper and lower $\mathrm{SU}(3)$ tensor indices. In the isospin invariant limit (where $\alpha_{1}=\alpha_{2}$ etc.), information will be obtained only for the kappa type particles (e.g. $\kappa^{+}=S_{1}^{3}$ when mixing is neglected). If isospin violation information is inserted, information may be obtained also about the isovector scalars like $a_{0}^{+}(980)$ (which is represented by $S_{1}^{2}$ when mixing is neglected). Next, let us differentiate successively the axial vector generating equation with respect to $\phi$ and to $\phi^{\prime}$. It is neater to write the results first for the case when fields with different upper and lower tensor indices are involved:

$$
\begin{aligned}
\left(\alpha_{a}+\alpha_{b}\right)\left\langle\frac{\partial^{2} V_{0}}{\partial \phi_{b}^{a} \partial \phi_{a}^{b}}\right\rangle+\left(\beta_{a}+\beta_{b}\right)\left\langle\frac{\partial^{2} V_{0}}{\partial{\phi^{\prime}}_{b}^{a} \partial \phi_{a}^{b}}\right\rangle=2\left(A_{a}+A_{b}\right), \\
\left(\alpha_{a}+\alpha_{b}\right)\left\langle\frac{\partial^{2} V_{0}}{\partial{\phi^{\prime}}_{b}^{a} \partial \phi_{a}^{b}}\right\rangle+\left(\beta_{a}+\beta_{b}\right)\left\langle\frac{\partial^{2} V_{0}}{\partial{\phi^{\prime}}_{b}^{a} \partial{\phi^{\prime}}_{a}^{b}}\right\rangle=0
\end{aligned}
$$

Next, let us write the corresponding equations for the case when the upper and lower tensor indices on each field are the same.

$$
\begin{aligned}
& \alpha_{b}\left\langle\frac{\partial^{2} V_{0}}{\partial \phi_{a}^{a} \partial \phi_{b}^{b}}\right\rangle+\beta_{b}\left\langle\frac{\partial^{2} V_{0}}{\partial \phi_{a}^{a} \partial{\phi^{\prime}}_{b}^{b}}\right\rangle=\sum_{g} \beta_{g}\left\langle\frac{\partial^{2} V_{0}}{\partial \phi_{a}^{a} \partial{\phi^{\prime}}_{g}^{g}}\right\rangle-\frac{8 c_{3}}{\alpha_{a}}, \\
& \alpha_{b}\left\langle\frac{\partial^{2} V_{0}}{\partial{\phi^{\prime}}_{a}^{\prime} \partial \phi_{b}^{b}}\right\rangle+\beta_{b}\left\langle\frac{\partial^{2} V_{0}}{\partial{\phi^{\prime}}_{a}^{a} \partial{\phi^{\prime}}_{b}^{b}}\right\rangle=\sum_{g} \beta_{g}\left\langle\frac{\partial^{2} V_{0}}{\partial{\phi^{\prime}}_{a}^{a} \partial{\phi^{\prime}}_{g}^{\prime}}\right\rangle .
\end{aligned}
$$

Note that the axial generating equation provides information on the masses of all the pseudoscalars. Further differentiations will relate a large number of trilinear and quadrilinear coupling constants to the meson masses and to the quark mass coefficients, $A_{a}$.

To fully characterize the system we will also require some knowledge of the axial vector and vector currents [38] obtained by Noether's method:

$$
\begin{aligned}
\left(J_{\mu}^{a x i a l}\right)_{a}^{b} & =\left(\alpha_{a}+\alpha_{b}\right) \partial_{\mu} \phi_{a}^{b}+\left(\beta_{a}+\beta_{b}\right) \partial_{\mu} \phi^{\prime}{ }_{a}^{b}+\cdots \\
\left(J_{\mu}^{v e c t o r}\right)_{a}^{b} & =i\left(\alpha_{a}-\alpha_{b}\right) \partial_{\mu} S_{a}^{b}+i\left(\beta_{a}-\beta_{b}\right) \partial_{\mu}{S^{\prime}}_{a}^{b}+\cdots
\end{aligned}
$$

where the three dots stand for terms bilinear in the fields. 


\section{PREDICTIONS FOR MASS SPECTRUM}

Here we consider the predictions for the mass spectrum of the model with the Lagrangian given in Eq. (13), whose potential contains any $\mathrm{SU}(3)_{L} \times \mathrm{SU}(3)_{R} \times \mathrm{U}(1)_{A}$ invariant terms whatsoever, amended with the $\mathrm{SU}(3)_{L} \times \mathrm{SU}(3)_{R}$ but not $U(1)_{A}$ invariant term of Eq. (18) as well as the term, Eq. 222) which transforms exactly like the QCD quark mass term. A characteristic feature is mixing between fields with the same quantum numbers. Specifically, there is information about mixing between $\pi$ and $\pi^{\prime}$, between $K$ and $K^{\prime}$, between $\kappa$ and $\kappa^{\prime}$ and among among the four $\eta$ type (isosinglet) states. We will take these up in turn. Note that we will be working in the isotopic spin invariant limit [46].

\section{A. The $\pi-\pi^{\prime}$ system}

For compactness let us denote,

$$
\begin{aligned}
& x_{\pi}=\frac{2 A_{1}}{\alpha_{1}}, \\
& y_{\pi}=\left\langle\frac{\partial^{2} V}{\partial{\phi^{\prime}}_{2}^{1} \partial{\phi^{\prime}}_{1}^{2}}\right\rangle, \\
& z_{\pi}=\frac{\beta_{1}}{\alpha_{1}} .
\end{aligned}
$$

Here we have introduced the total potential $V=V_{0}+V_{S B}$. However, since the second derivatives of $V_{S B}$ vanish with our present choice of flavor symmetry breaker we may just use $V_{0}$. Substituting $a=1, b=2$ into both of Eqs. (32) enables us to write the (non-diagonal) matrix of squared $\pi$ and $\pi^{\prime}$ masses as:

$$
\left(M_{\pi}^{2}\right)=\left[\begin{array}{cc}
x_{\pi}+z_{\pi}^{2} y_{\pi} & -z_{\pi} y_{\pi} \\
-z_{\pi} y_{\pi} & y_{\pi}
\end{array}\right]
$$

It is clear that $z_{\pi}$ is a measure of the mixing between $\pi$ and $\pi^{\prime}$ since the matrix becomes diagonal in the limit when $z_{\pi}$ is set to zero. So we see that $x_{\pi}$ would be the squared pion mass in the single $\mathrm{M}$ model and $y_{\pi}$ represents the squared mass of the "bare" $\pi^{\prime}$. Denoting the eigenvalues of this matrix by $m_{\pi}^{2}$ and $m_{\pi^{\prime}}^{2}$, we read off the product and sum rules:

$$
\begin{array}{r}
m_{\pi}^{2} m_{\pi^{\prime}}^{2}=x_{\pi} y_{\pi}, \\
m_{\pi}^{2}+m_{\pi^{\prime}}^{2}=x_{\pi}+y_{\pi}\left(1+z_{\pi}^{2}\right) .
\end{array}
$$

Assuming that the values of $m_{\pi}$ and $m_{\pi^{\prime}}$ are known, the first of these equations expresses $y_{\pi}$ in terms of $x_{\pi}$. Then the second of these equations also expresses $z_{\pi}^{2}$ in terms of $x_{\pi}$. The value of $x_{\pi}$ is not known but its range is restricted to be,

$$
m_{\pi}^{2} \leq x_{\pi} \leq m_{\pi^{\prime}}^{2}
$$

This range may be derived by expressing $z_{\pi}^{2}$ in terms of $x_{\pi}$ as mentioned and requiring $z_{\pi}^{2} \geq 0$.

The transformation between the diagonal fields (say $\pi^{+}$and $\pi^{\prime+}$ ) and the original pion fields is defined as:

$$
\left[\begin{array}{c}
\pi^{+} \\
\pi^{\prime+}
\end{array}\right]=\left[\begin{array}{cc}
\cos \theta_{\pi} & -\sin \theta_{\pi} \\
\sin \theta_{\pi} & \cos \theta_{\pi}
\end{array}\right]\left[\begin{array}{c}
\phi_{1}^{2} \\
\phi_{1}^{\prime 2}
\end{array}\right] .
$$

The explicit diagonalization gives an expression for the mixing angle $\theta_{\pi}$ :

$$
\tan \left(2 \theta_{\pi}\right)=\frac{-2 y_{\pi} z_{\pi}}{y_{\pi}\left(1-z_{\pi}^{2}\right)-x_{\pi}},
$$

which evidently is also known, up to a sign choice for $z_{\pi}$, once $x_{\pi}$ is specified.

The mixing angle, $\theta_{\pi}$ can also be connected to the experimentally known value of the pion decay constant (i.e. the amplitude for the $\pi^{+}$meson to decay to two leptons). Substituting the expressions from Eq. (39) for $\phi_{1}^{2}$ and ${\phi^{\prime}}_{1}^{2}$ in terms of the physical fields $\pi^{+}$and $\pi^{\prime+}$ into Eq. (34) yields,

$$
\begin{aligned}
\left(J_{\mu}^{\text {axial }}\right)_{1}^{2} & =F_{\pi} \partial_{\mu} \pi^{+}+F_{\pi^{\prime}} \partial_{\mu} \pi^{\prime+}+\cdots \\
F_{\pi} & =\left(\alpha_{1}+\alpha_{2}\right) \cos \theta_{\pi}-\left(\beta_{1}+\beta_{2}\right) \sin \theta_{\pi}, \\
F_{\pi^{\prime}} & =\left(\alpha_{1}+\alpha_{2}\right) \sin \theta_{\pi}+\left(\beta_{1}+\beta_{2}\right) \cos \theta_{\pi} .
\end{aligned}
$$


We can then obtain $\alpha_{1}$ (in the isospin invariant limit) as,

$$
\alpha_{1}=\frac{F_{\pi}}{2\left(\cos \theta_{\pi}-z_{\pi} \sin \theta_{\pi}\right)} .
$$

We then successively obtain $A_{1}$ from the definition of $x_{\pi}$, Eq. (35) and $\beta_{1}$ from the definition of $z_{\pi}$, Eq, (35). To sum up, specifying $x_{\pi}$ and the experimental quantities $m_{\pi}, m_{\pi^{\prime}}$ and $F_{\pi}$ determines all the other parameters of the $\pi-\pi^{\prime}$ system.

\section{B. The $K-K^{\prime}$ system}

The treatment of this system is almost exactly analogous to that of the $\pi-\pi^{\prime}$ system above when one defines the analogous variables,

$$
\begin{aligned}
& x_{K}=\frac{2\left(A_{3}+A_{1}\right)}{\alpha_{3}+\alpha_{1}}, \\
& y_{K}=\left\langle\frac{\partial^{2} V}{\partial \phi^{\prime 1}{ }_{3}^{\prime} \phi_{1}^{\prime 3}}\right\rangle, \\
& z_{K}=\frac{\beta_{3}+\beta_{1}}{\alpha_{3}+\alpha_{1}} .
\end{aligned}
$$

Substituting $a=1, b=3$ into both of Eqs. (32) enables us to write the (non-diagonal) matrix of squared $K$ and $K^{\prime}$ masses as:

$$
\left(M_{K}^{2}\right)=\left[\begin{array}{cc}
x_{K}+z_{K}^{2} y_{K} & -z_{K} y_{K} \\
-z_{K} y_{K} & y_{K}
\end{array}\right]
$$

This is observed to be identical to the expression for $\left(M_{\pi}^{2}\right)$ in Eq. (36) when one simply substitutes everywhere $K$ for $\pi$ and $K^{\prime}$ for $\pi^{\prime}$. Similarly, the four equations (37), (38), (39) and (40) continue to hold when one substitutes everywhere $K$ for $\pi$ and $K^{\prime}$ for $\pi^{\prime}$. Similarly, the $K^{+}$decay constant, $F_{K}$ is now defined from,

$$
\begin{aligned}
\left(J_{\mu}^{\text {axial }}\right)_{1}^{3} & =F_{K} \partial_{\mu} K^{+}+F_{K^{\prime}} \partial_{\mu} K^{\prime+}+\cdots, \\
F_{K} & =\left(\alpha_{1}+\alpha_{3}\right) \cos \theta_{K}-\left(\beta_{1}+\beta_{3}\right) \sin \theta_{K}, \\
F_{K^{\prime}} & =\left(\alpha_{1}+\alpha_{3}\right) \sin \theta_{K}+\left(\beta_{1}+\beta_{3}\right) \cos \theta_{K} \cdot
\end{aligned}
$$

We can then obtain $\alpha_{3}+\alpha_{1}$ (in the isospin invariant limit) as,

$$
\alpha_{3}+\alpha_{1}=\frac{F_{K}}{\cos \theta_{K}-z_{K} \sin \theta_{K}} .
$$

We then successively obtain $A_{3}+A_{1}$ from the definition of $x_{K}$ and $\beta_{3}+\beta_{1}$ from the definition of $z_{K}$. To sum up, specifying $x_{K}$ and the experimental quantities $m_{K}, m_{K^{\prime}}$ and $F_{K}$ determines all the other parameters of the $K-K^{\prime}$ system.

\section{The $\kappa-\kappa^{\prime}$ system}

Again, we can treat this system in an exactly analogous way to the $\pi-\pi^{\prime}$ and $K-K^{\prime}$ cases if we define the analogous quantities:

$$
\begin{aligned}
& x_{\kappa}=\frac{2\left(A_{3}-A_{1}\right)}{\alpha_{3}-\alpha_{1}}, \\
& y_{\kappa}=\left\langle\frac{\partial^{2} V}{\partial S_{3}^{\prime 1} \partial S_{1}^{\prime 3}}\right\rangle, \\
& z_{\kappa}=\frac{\beta_{3}-\beta_{1}}{\alpha_{3}-\alpha_{1}} .
\end{aligned}
$$


In this case, however, the vector generating equations in Eqs. (31) with the choices $a=1$ and $b=3$ are used. The transformation between the diagonal and original strange scalar fields is given by,

$$
\left[\begin{array}{c}
\kappa^{+} \\
\kappa^{\prime+}
\end{array}\right]=\left[\begin{array}{cc}
\cos \theta_{\kappa} & -\sin \theta_{\kappa} \\
\sin \theta_{\kappa} & \cos \theta_{\kappa}
\end{array}\right]\left[\begin{array}{c}
S_{1}^{3} \\
S_{1}^{\prime 3}
\end{array}\right],
$$

where the mixing angle is determined by the diagonalization:

$$
\tan \left(2 \theta_{\kappa}\right)=\frac{-2 y_{\kappa} z_{\kappa}}{y_{\kappa}\left(1-z_{\kappa}^{2}\right)-x_{\kappa}} .
$$

We may define $\kappa$ "decay constants' as,

$$
\begin{aligned}
F_{\kappa} & =\left(\alpha_{3}-\alpha_{1}\right) \cos \theta_{\kappa}-\left(\beta_{3}-\beta_{1}\right) \sin \theta_{\kappa}, \\
F_{\kappa^{\prime}} & =\left(\alpha_{3}-\alpha_{1}\right) \sin \theta_{\kappa}+\left(\beta_{3}-\beta_{1}\right) \cos \theta_{\kappa},
\end{aligned}
$$

although there is no direct experimental information available about them.

Now let us consider the $\pi-\pi^{\prime}, K-K^{\prime}$ and $\kappa-\kappa^{\prime}$ systems together. Using the first two we can get all of $A_{1}, A_{3}, \alpha_{1}, \alpha_{3}, \beta_{1}, \beta_{3}$ from the experimental masses of $\pi, \pi^{\prime}, K, K^{\prime}$, the experimental decay constants $F_{\pi}, F_{K}$ and the assumed values of $x_{\pi}$ and $x_{K}$, as seen above. This means that $x_{\kappa}$ and $z_{\kappa}$ may be read off directly from Eqs. (47) while $y_{\kappa}$ can be found from the product rule $m_{\kappa}^{2} m_{\kappa^{\prime}}^{2}=x_{\kappa} y_{\kappa}$ if $m_{\kappa}$ and $m_{\kappa^{\prime}}$ are furnished. Thus all the parameters of the $\kappa-\kappa^{\prime}$ system are known, given the input masses and the values of $x_{\pi}$ and $x_{K}$. However we have not yet made use of the sum rule analogous to the second of Eqs. (37). This provides another way to calculate $z_{\kappa}$ so we get the consistency condition:

$$
\left(\frac{\beta_{3}-\beta_{1}}{\alpha_{3}-\alpha_{1}}\right)^{2}=\frac{x_{\kappa}\left(m_{\kappa}^{2}+m_{\kappa^{\prime}}^{2}-x_{\kappa}\right)}{m_{\kappa}^{2} m_{\kappa^{\prime}}^{2}}-1
$$

Since the quantities in this equation depend on both $x_{\pi}$ and $x_{K}$, the solution can determine the value of $x_{K}$ for each choice of $x_{\pi}$. In other words, if $x_{\pi}$ is specified, the parameters of the $\pi-\pi^{\prime}$, the $K-K^{\prime}$ and the $\kappa-\kappa^{\prime}$ systems are all determined in the present model.

\section{The $\eta$ system}

This system is more complicated because, even in the isotopic spin invariant limit, there are four different $I=0$ pseudoscalars which can mix with each other. These may be put together as a column vector according to,

$$
\Phi_{0}=\left[\begin{array}{c}
\frac{\phi_{1}^{1}+\phi_{2}^{2}}{\sqrt{2}} \\
\phi_{3}^{3} \\
\frac{\phi_{1}^{\prime}{ }_{1}^{\prime} \phi_{2}^{2}}{\sqrt{2}} \\
\phi_{3}^{\prime 3}
\end{array}\right] .
$$

The part of the Lagrangian describing the masses of the $I=0$ pseudoscalars is then: $\mathcal{L}=-(1 / 2) \Phi_{0}^{T}\left(M_{\eta}^{2}\right) \Phi_{0}$, where $\left(M_{\eta}^{2}\right)$ is a symmetric $4 \times 4$ matrix. Relations among the matrix elements follow by using both of Eqs. (33). These connect the transition masses both to the "bare" unprimed particle masses and to the "bare" primed particle masses. The use of isospin invariance relations like the ones given in Appendix B may also be useful. Eventually, the matrix elements of $\left(M_{\eta}^{2}\right)$ depend on four new quantities in addition to the ones appearing in the above three subsystems. The resulting matrix elements are listed below:

$$
\begin{aligned}
\left(M_{\eta}^{2}\right)_{11}= & \frac{2 A_{1}}{\alpha_{1}}-\frac{16 c_{3}}{\alpha_{1}^{2}}-\frac{\beta_{1}^{2} m_{\pi}^{2} m_{\pi^{\prime}}^{2}}{2 A_{1} \alpha_{1}}+2\left(\frac{\beta_{1}}{\alpha_{1}}\right)^{2}\left\langle\frac{\partial^{2} V}{\left(\partial{\phi^{\prime}}_{1}^{1}\right)^{2}}\right\rangle+4\left(\frac{\beta_{1} \beta_{3}}{\alpha_{1}^{2}}\right)\left\langle\frac{\partial^{2} V}{\partial{\phi^{\prime}}_{1}^{1} \partial{\phi^{\prime}}_{3}^{3}}\right\rangle \\
& +2\left(\frac{\beta_{3}}{\alpha_{1}}\right)^{2}\left\langle\frac{\partial^{2} V}{\partial{\phi^{\prime}}_{3}^{3} \partial \phi_{3}^{3}}\right\rangle \\
\left(M_{\eta}^{2}\right)_{12}= & -\frac{8 \sqrt{2} c_{3}}{\alpha_{1} \alpha_{3}}-\frac{\beta_{1}^{2} m_{\pi}^{2} m_{\pi^{\prime}}^{2}}{\sqrt{2} A_{1} \alpha_{3}}+\left(\frac{2 \sqrt{2} \beta_{1}^{2}}{\alpha_{1} \alpha_{3}}\right)\left\langle\frac{\partial^{2} V}{\left(\partial{\phi^{\prime}}_{1}^{1}\right)^{2}}\right\rangle+\left(\frac{2 \sqrt{2} \beta_{1} \beta_{3}}{\alpha_{1} \alpha_{3}}\right)\left\langle\frac{\partial^{2} V}{\partial{\phi^{\prime}}_{1}^{1} \partial{\phi^{\prime}}_{3}^{3}}\right\rangle
\end{aligned}
$$




$$
\begin{aligned}
& \left(M_{\eta}^{2}\right)_{13}=-\frac{\beta_{1} m_{\pi}^{2} m_{\pi^{\prime}}^{2}}{2 A_{1}}+2\left(\frac{\beta_{1}}{\alpha_{1}}\right)\left\langle\frac{\partial^{2} V}{\left(\partial{\phi^{\prime}}^{1}\right)^{2}}\right\rangle+2\left(\frac{\beta_{3}}{\alpha_{1}}\right)\left\langle\frac{\partial^{2} V}{\partial{\phi^{\prime}}_{1}^{\prime} \partial{\phi^{\prime}}_{3}^{3}}\right\rangle \\
& \left(M_{\eta}^{2}\right)_{14}=\left(\frac{\sqrt{2} \beta_{1}}{\alpha_{1}}\right)\left\langle\frac{\partial^{2} V}{\partial{\phi^{\prime}}_{1}^{1} \partial{\phi_{3}^{\prime}}_{3}^{3}}\right\rangle+\left(\frac{\sqrt{2} \beta_{3}}{\alpha_{1}}\right)\left\langle\frac{\partial^{2} V}{\partial{\phi^{\prime}}_{3}^{3} \partial{\phi^{\prime}}_{3}^{3}}\right\rangle \\
& \left(M_{\eta}^{2}\right)_{22}=\frac{2 A_{3}}{\alpha_{3}}-\frac{8 c_{3}}{\alpha_{3}^{2}}-\frac{\alpha_{1} \beta_{1}^{2} m_{\pi}^{2} m_{\pi^{\prime}}^{2}}{A_{1} \alpha_{3}^{2}}+4\left(\frac{\beta_{1}}{\alpha_{3}}\right)^{2}\left\langle\frac{\partial^{2} V}{\left(\partial \phi^{\prime 1}{ }_{1}^{2}\right)^{2}}\right\rangle \\
& \left(M_{\eta}^{2}\right)_{23}=-\frac{\alpha_{1} \beta_{1} m_{\pi}^{2} m_{\pi^{\prime}}^{2}}{\sqrt{2} A_{1} \alpha_{3}}+\left(\frac{2 \sqrt{2} \beta_{1}}{\alpha_{3}}\right)\left\langle\frac{\partial^{2} V}{\left(\partial \phi_{1}^{\prime}\right)^{2}}\right\rangle \\
& \left(M_{\eta}^{2}\right)_{24}=\left(\frac{2 \beta_{1}}{\alpha_{3}}\right)\left\langle\frac{\partial^{2} V}{\partial \phi_{1}^{\prime} \partial \phi_{3}^{\prime 3}}\right\rangle \\
& \left(M_{\eta}^{2}\right)_{33}=-\frac{\alpha_{1} m_{\pi}^{2} m_{\pi^{\prime}}^{2}}{2 A_{1}}+2\left\langle\frac{\partial^{2} V}{\left(\partial{\phi^{\prime}}_{1}^{1}\right)^{2}}\right\rangle \\
& \left(M_{\eta}^{2}\right)_{34}=\sqrt{2}\left\langle\frac{\partial^{2} V}{\partial{\phi^{\prime}}_{1}^{1} \partial{\phi^{\prime}}_{3}^{3}}\right\rangle \\
& \left(M_{\eta}^{2}\right)_{44}=\left\langle\frac{\partial^{2} V}{\partial{\phi^{\prime}}_{3}^{3} \partial{\phi^{\prime}}_{3}^{3}}\right\rangle
\end{aligned}
$$

The four new quantities are $c_{3}$, discussed earlier, and the "bare" primed squared masses:

$$
\left\langle\frac{\partial^{2} V}{\left(\partial{\phi^{\prime}}^{1}\right)^{2}}\right\rangle, \quad\left\langle\frac{\partial^{2} V}{\partial{\phi^{\prime}}_{1}^{1} \partial{\phi^{\prime}}_{3}^{3}}\right\rangle, \quad\left\langle\frac{\partial^{2} V}{\partial{\phi^{\prime}}_{3}^{3} \partial{\phi^{\prime}}_{3}^{3}}\right\rangle .
$$

These four quantities may be found by inputing the masses of four isosinglet pseudoscalars. The net result is that all four systems discussed will be completely described if all the experimental masses and the decay constants, $F_{\pi}, F_{K}$ are specified together with an assumed value for $x_{\pi}$.

\section{COMPARISON WITH EXPERIMENT AND DISCUSSION}

In the preceding section we gave the tree level formulas resulting from the $M-M^{\prime}$ model with any $\mathrm{SU}(3)_{L} \times$ $\mathrm{SU}(3)_{R} \times \mathrm{U}(1)_{A}$ invariant terms together with a single "instanton" type term which mocks up the $\mathrm{U}(1)_{A}$ anomaly and the simplest structure which mocks up the quark mass terms. Isotopic spin invariance was also assumed. Information is provided for only the pseudoscalar nonets and the strange scalar particles. Information about the scalar isotriplets can be obtained by including isospin violation effects while information about the scalar isosinglets requires either assuming some specific form for the invariant interaction terms or computing other physical quantities. These will be discussed elsewhere. Now we will input the experimental masses to try to learn what the model has to say about the quark structure of the various mesons being described. In particular we are interested in the mixing angles like $\theta_{\pi}$, governing admixtures of $q \bar{q}$ and $q q \bar{q} \bar{q}$ in the physical states and the four quark "condensate" strengths, $\beta_{a}$ which are associated with this mixing in the present model.

The well known lowest pseudoscalar nonet masses and decay constants will be taken, for definiteness (considering the ambiguity as to which member of a non trivial isospin multiplet to choose), to be:

$$
\begin{gathered}
m_{\pi}=0.137 \mathrm{GeV}, \quad m_{K}=0.496 \mathrm{GeV}, \\
m_{\eta}=0.548 \mathrm{GeV}, \quad m_{\eta^{\prime}}=0.958 \mathrm{GeV}, \\
F_{\pi}=0.131 \mathrm{GeV}, \quad F_{K}=0.160 \mathrm{GeV} .
\end{gathered}
$$

Next, let us consider what are the suitable experimental inputs for the masses of the excited mesons, $\pi^{\prime}, K^{\prime} \cdot \kappa^{\prime}$ and for the $\kappa$ meson itself. In the latest Review of particle properties [47] there are two dotted (i.e. considered established) candidates for excited pions below $2 \mathrm{GeV}$ : the $\pi(1300)$ and the $\pi(1800)$. These particles could have four quark components and/or radially excited two quark components. In fact, judging from an investigation of excited baryons [48], it is likely that both types are present. Clearly, however, for our present investigation it seems reasonable to assume that the four quark component is the dominant one and to choose the lower mass object as the more suitable one. Similarly there are two undotted (non established) excited kaon candidates: the K(1460) and the $\mathrm{K}(1830)$. We will again choose the lower value. As candidates for an excited strange scalar there is a dotted $K_{0}^{*}(1430)$ 
and an undotted $K_{0}^{*}(1950)$ and we again choose the lower value. In the case of the low mass strange scalar there is an undotted $K_{0}^{*}(800)$ candidate, which we will interpret, with the help of [15], to be closer to $900 \mathrm{MeV}$. We summarize these choices:

$$
\begin{array}{cl}
m_{\pi^{\prime}}=1.30 \mathrm{GeV}, & m_{K^{\prime}}=1.46 \mathrm{GeV}, \\
m_{\kappa}=0.90 \mathrm{GeV}, & m_{\kappa^{\prime}}=1.42 \mathrm{GeV} .
\end{array}
$$

For the excited $\eta$ type pseudoscalar particles the Review of particle properties lists, below 2 GeV, the possible masses (all in $\mathrm{GeV})$ :

$$
1.294, \quad 1.410, \quad 1.476, \quad 1.760 .
$$

The first three of these are dotted but the fourth is undotted. Here it seems more difficult to a priori choose which are most relevant so we shall study all possible pairings in a systematic way.

First let us discuss the $\pi-\pi^{\prime}, K-K^{\prime}$ and $\kappa-\kappa^{\prime}$ systems. After using the inputs of Eq. (55), all features of these systems in our model will, as already discussed, be determined by specifying $x_{\pi}$. Table shows the predicted physical parameters for three values of $x_{\pi}$. For orientation we note that in the chiral model with a single field, M one has

$$
\begin{gathered}
\alpha_{1} \rightarrow F_{\pi} / 2=0.0655 \mathrm{GeV}, \alpha_{3} \rightarrow F_{K}-\alpha_{1}=0.0945 \mathrm{GeV}, \\
A_{1} \rightarrow \frac{\alpha_{1}}{2} m_{\pi}^{2}=6.15 \times 10^{-4} \mathrm{GeV}^{3}, A_{3} \rightarrow \frac{F_{K}}{2} m_{K}^{2}=0.01866 \mathrm{GeV}^{3}, \\
\beta_{1} \rightarrow 0 \quad, \beta_{2} \rightarrow 0 .
\end{gathered}
$$

The single $\mathrm{M}$ model corresponds to the choice $x_{\pi}=m_{\pi}^{2}$. Increasing $x_{\pi}$ has the effect of increasing the admixture of the "four quark" field component in the physical pion. The "quark mass ratio", $A_{3} / A_{1}=30.3$ in the single M

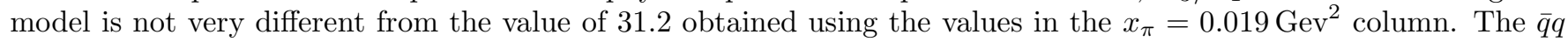
meson condensates $\alpha_{1}$ and $\alpha_{3}$ are also very similar. Of course the "four quark" meson condensates $\beta_{1}$ amd $\beta_{2}$ are zero without $M^{\prime}$. Despite the similarities, the $6.4^{\circ}$ mixing angle already corresponds to about an 11 percent "four quark" admixture in the physical pion wave function. Considering that the accuracy of current algebra predictions for low energy pion physics is roughly ten percent, it seems that this choice of $x_{\pi}$ is the most plausible one. One sees from the second and third columns that relatively small increases in $x_{\pi}$ lead to large increases in four quark admixture for the pion and the kaon. Interestingly, the behavior of the four quark admixture in the strange scalar meson $\kappa$ is quite different. When the pseudoscalars are closer to pure "two quark" states in the model the scalar has a large four quark admixture $\left(34.1^{\circ}\right.$, with the choice of $x_{\pi}$ in the first column). Thus the result is consistent with having a fairly large four quark component in the light scalars.

\begin{tabular}{c||c|c|c}
\hline \hline & $x_{\pi}=0.019\left(\mathrm{GeV}^{2}\right)$ & $x_{\pi}=0.021\left(\mathrm{GeV}^{2}\right)$ & $x_{\pi}=0.022\left(\mathrm{GeV}^{2}\right)$ \\
\hline \hline$\theta_{\pi}$ (deg.) & -6.4 & -19.1 & -22.7 \\
\hline$\theta_{k}$ (deg.) & -11.2 & -22.9 & -26.2 \\
\hline$\theta_{\kappa}$ (deg.) & 34.1 & 28.1 & 26.5 \\
\hline$A_{1}\left(\mathrm{GeV}^{3}\right)$ & $6.19 \times 10^{-4}$ & $6.51 \times 10^{-4}$ & $6.66 \times 10^{-4}$ \\
\hline$A_{3}\left(\mathrm{GeV}^{3}\right)$ & $1.94 \times 10^{-2}$ & $2.07 \times 10^{-2}$ & $2.12 \times 10^{-2}$ \\
\hline$\alpha_{1}(\mathrm{GeV})$ & $6.51 \times 10^{-2}$ & $6.20 \times 10^{-2}$ & $6.06 \times 10^{-2}$ \\
\hline$\alpha_{3}(\mathrm{GeV})$ & $9.24 \times 10^{-2}$ & $8.83 \times 10^{-2}$ & $8.69 \times 10^{-2}$ \\
\hline$\beta_{1}(\mathrm{GeV})$ & $7.18 \times 10^{-3}$ & $2.12 \times 10^{-2}$ & $2.50 \times 10^{-2}$ \\
\hline$\beta_{3}(\mathrm{GeV})$ & $2.03 \times 10^{-2}$ & $3.38 \times 10^{-2}$ & $3.74 \times 10^{-2}$ \\
\hline \hline
\end{tabular}

TABLE I: $\theta_{\pi}, \theta_{K}$ and $\theta_{\kappa}$ are respectively the "four quark" admixtures in the $\pi, K$ and $\kappa$ states. $A_{1}, A_{3}$ represent the quark mass parameters while $\alpha_{1}, \alpha_{3}$ and $\beta_{1}, \beta_{3}$ represent respectively the two and four quark condensate strengths. These are plotted as functions of the assumed "bare" pion squared mass, $x_{\pi}$.

The analogs of the two quark condensates $\alpha_{1}=\alpha_{2}$ and $\alpha_{3}$ are approximately equal, in agreement with the usual assumption that the vacuum is approximately $\mathrm{SU}(3)$ symmetric. The analogs of the four quark condensates in this model are roughly an order of magnitude smaller than the similarly normalized two quark condensates. They are furthermore seen to deviate appreciably from SU(3) symmetry. It should be noted, as discussed in ref, 16 for example, that the tensor indices for the primed mesons really correspond to "dual quark" or diquark indices in accordance with,

$$
Q_{a} \sim \epsilon_{a b c} \bar{q}^{b} \bar{q}^{c}
$$


Thus in terms of the usual quarks,

$$
\beta_{1} \sim\langle\bar{d} d \bar{s} s\rangle, \quad \beta_{2} \sim\langle\bar{u} u \bar{s} s\rangle, \quad \beta_{1} \sim\langle\bar{d} d \bar{u} u\rangle .
$$

Now consider the mixing of the four $\eta$ type fields in the model. The basis is given in Eq. (52) while the elements of the $4 \times 4$ mass squared matrix are given in Eq.(53). The orthogonal transformation matrix, $K$ which relates the mass eigenstate fields, $\Phi$ to the original ones is defined by

$$
\Phi_{0}=K \Phi .
$$

As discussed in the previous section, there are, after using the symmetry information, four new unknown parameters characterizing the $\eta$ system. Thus taking the four mass eigenvalues from experiment could in principle determine, together with results from the $\pi-\pi^{\prime}, K=K^{\prime}$ and $\kappa-\kappa^{\prime}$ systems, everything about the $\eta$ system for a given value of $x_{\pi}$. However there is no guarantee that there will be an exact solution for all choices of experimental parameters. This is the case, in fact, so we will search numerically for a choice of "theoretical" masses which will best fit the experimental inputs. The criterion for goodness of fit will be taken to be the smallness of the quantity:

$$
\chi \equiv \sum_{i}\left|m_{i}^{\text {exp. }}-m_{i}^{\text {theo. }}\right| / m_{i}^{\text {exp. }}
$$

As shown in Eq. (57), there are three established candidates and one not yet established candidate below $2 \mathrm{GeV}$ for the two excited $\eta$ states. This yields six possible scenarios for choosing them. The quantity $\chi$ for each choice is shown in Table II for three values of the parameter $x_{\pi}$. It may be observed that the fits typically get worse with increasing $x_{\pi}$, so it is reasonable to consider the choice $0.019 \mathrm{GeV}^{2}$ for this quantity as we did previously. The smallest values of $\chi$ are found for scenarios 5 and 6 . However these both involve the $\eta(1760)$ state which is the one not yet established. The smallest value of $\chi$ using only established states is scenario 2 . This case corresponds to an exact fit with eta type masses in $\mathrm{GeV}$ (experimental values in parentheses for comparison):

$$
\begin{array}{ll}
0.533(0.548), & 0.963(0.958), \\
1.327(1.294), & 1.716(1.476) .
\end{array}
$$

\begin{tabular}{c||c|c|c}
\hline \hline Scenario & $x_{\pi}=0.019\left(\mathrm{GeV}^{2}\right)$ & $x_{\pi}=0.021\left(\mathrm{GeV}^{2}\right)$ & $x_{\pi}=0.022\left(\mathrm{GeV}^{2}\right)$ \\
\hline \hline $1:\{\eta(1295), \eta(1405)\}$ & $6.23 \times 10^{-2}$ & $3.99 \times 10^{-1}$ & $5.08 \times 10^{-1}$ \\
\hline $2:\{\eta(1295), \eta(1475)\}$ & $2.85 \times 10^{-2}$ & $3.39 \times 10^{-1}$ & $4.44 \times 10^{-1}$ \\
\hline $3:\{\eta(1295), \eta(1760)\}$ & $2.35 \times 10^{-2}$ & $1.37 \times 10^{-1}$ & $2.28 \times 10^{-1}$ \\
\hline $4:\{\eta(1405), \eta(1475)\}$ & $8.28 \times 10^{-2}$ & $3.63 \times 10^{-1}$ & $4.49 \times 10^{-1}$ \\
\hline $5:\{\eta(1405), \eta(1760)\}$ & $1.50 \times 10^{-2}$ & $1.62 \times 10^{-1}$ & $2.38 \times 10^{-1}$ \\
\hline $6:\{\eta(1475), \eta(1760)\}$ & $2.84 \times 10^{-2}$ & $1.78 \times 10^{-1}$ & $2.68 \times 10^{-1}$ \\
\hline \hline
\end{tabular}

TABLE II: A goodness of fit quantity, $\chi \equiv \sum_{i}\left|m_{i}^{\text {exp. }}-m_{i}^{\text {theo. }}\right| / m_{i}^{\text {exp. }}$, where the $m_{i}$ are the four mass eigenvalues of the $\eta$ type fields, is given for 6 possible scenarios and for three values of $x_{\pi}$. Each scenario corresponds to a choice of $\eta$ type fields including the $\eta(548)$ and the $\eta(958)$ as well as the two listed in the left hand column.

The detailed content of all the $\eta$ mass eigenstates can be read off from the matrix $K^{-1}$. For scenario 2 we have,

$$
K^{-1}=\left[\begin{array}{cccc}
-0.570 & 0.750 & -0.023 & 0.333 \\
-0.329 & -0.573 & 0.142 & 0.737 \\
0.704 & 0.267 & -0.309 & 0.581 \\
0.267 & 0.192 & 0.940 & 0.088
\end{array}\right]
$$

Thus, in the present model there is an 89 percent probability $\left(\left(K^{-1}\right)_{11}^{2}+\left(K^{-1}\right)_{12}^{2}\right)$ that the $\eta(548)$ is a quark-antiquark state and an eleven percent probability that it is a four quark state. As expected, the $\eta(548)$ is most likely to be in an $\bar{s} s$ state. In the case of the $\eta(958)$, there is a 44 percent probability for it to be in a quark antiquark state. There is a 54 percent probability for it to be in the four quark state $\phi_{3}^{\prime 3}$. This situation has some plausibility since in terms of ordinary quarks, the latter state has the content $\bar{u} u \bar{d} d$ and it should be most energetically favorable to bind a four quark state made without strange quarks. 
The other scenarios which don't employ the unconfirmed $\eta(1760)$ state (numbers 1 and 4 ) have contents very similar to the one in Eq. (64). On the other hand the three scenarios employing the $\eta(1760)$ have a rather different content, which seems unusual: scenarios 3,5 and 6 make the $\eta(958)$ almost completely $\phi_{3}^{\prime 3}$.

In scenario 2 , which seems the most reasonable choice, we notice that the $\eta(1295)$ has a 43 percent probability of being in a four quark state while the $\eta(1475)$ has an 89 percent probability of being in a four quark state. To sum up, the value $x_{\pi}=0.019 \mathrm{GeV}^{2}$ leads to fairly small four quark content in the light pseudoscalars- $\pi, K, \eta$ at the same time that the light scalar $\kappa$ has an appreciable four quark component. The "excited" $\eta$ 's are predominantly four quark states. The $\eta(960)$ is mainly two quark in content but has a non trivial four quark piece.

The results obtained here provide supporting evidence for the feature, illustrated in the first treatment of this model 29], that the lightest scalars, unlike the lightest pseudoscalars, have appreciable four quark components. That model neglected quark masses and used the simplified choice of terms shown in Eq. (14). The more recent treatment of ref. [42], includes two additional invariant terms beyond those in Eq. (14) (although not all the renormalizable terms shown in Appendix A) as well as four types of quark mass splitting terms. Our results for the present treatment, where quark masses are included and which holds for any possible $\mathrm{SU}(3)_{L} \times \mathrm{SU}(3)_{R} \times \mathrm{U}(1)_{A}$ conserving terms, are also in qualitative agreement for the $\pi$-type, K-type, $\eta$-type and $\kappa$-type states with that treatment. Roughly, this may be expected since the present approach includes any choice of invariant terms. However, we only used here the single quark mass splitting term of Eq. (22). Thus the results seem qualitatively robust with respect to the treatment of the mass splittings.

An interesting feature of our model is the presence of "four quark" condensates as signaled by the non-zero values of the $\beta_{a}$. To make a rough estimate of what this corresponds to in quark language we proceed as follows. In ref. [40] it was pointed out that the mass formulas of the single M linear sigma model could be transformed to the "current algebra" ones [49] by the replacements:

$$
A_{a}=m_{a} \Lambda^{2}, \quad \alpha_{a}=-\frac{\left\langle\bar{q}_{a} q_{a}\right\rangle}{2 \Lambda^{2}},
$$

where the $m_{a}$ are the ("current" type) quark masses and $\Lambda$ is the QCD scale factor. Taking $A_{1}=6.19 \times 10^{-4} \mathrm{GeV}^{2}$ from the left column of Table $\llbracket$ and $m_{1} \approx 5 \mathrm{MeV}$ we get $\Lambda \approx 0.35 \mathrm{GeV}$ (and $\left\langle\bar{q}_{a} q_{a}\right\rangle \approx-0.016 \mathrm{GeV}^{3}$ ). In the case of the four quark condensate, as one sees from the discussion in the Introduction, there are several ways to couple the four quarks together to make scalars. We are assuming that one such way has been selected. For that case, it is reasonable to expect, on dimensional grounds, that

$$
|\langle\bar{d} d \bar{s} s\rangle| \sim \Lambda^{5} \beta_{1} \approx 4 \times 10^{-5} \mathrm{GeV}^{6} .
$$

In comparing the scalar masses with experiment there are expected to be, as discussed in the first four sections of ref. [29], non-negligible corrections due to the use of unitary models for the pseudoscalar- pseudoscalar scattering based on this Lagrangian. We plan to report on this elsewhere. This should also enable us to study the isosinglet scalar masses. For both isosinglet scalars and pseudoscalars, the inclusion of possible glueball states is another interesting topic we plan to pursue. The additional symmetry breaking terms like those in Eqs. (19) and (23) seem also to be worth investigating.

\section{Acknowledgments}

We are happy to thank A. Abdel-Rehim, D. Black, M. Harada, S. Moussa, S. Nasri and F. Sannino for many helpful related discussions. The work of A.H.F. has been supported by the 2004 Crouse Grant from the School of Arts and Sciences, SUNY Institute of Technolgy. The work of R.J. and J.S. is supported in part by the U. S. DOE under Contract no. DE-FG-02-85ER 40231.

\section{APPENDIX A: RENORMALIZABLE MODEL}

The twenty one $\mathrm{SU}(3) \times \mathrm{SU}(3)$ invariant renormalizable terms in $V_{0}$ of Eq. (13) which can be made out of $M$ and $M^{\prime}$ are:

$$
\begin{aligned}
V_{0}= & -c_{2} \operatorname{Tr}\left(M M^{\dagger}\right)+\tilde{c}_{3}(\operatorname{det} M+\text { h.c. })+c_{4}^{a} \operatorname{Tr}\left(M M^{\dagger} M M^{\dagger}\right)+c_{4}^{b}\left(\operatorname{Tr}\left(M^{\dagger}\right)\right)^{2} \\
& +d_{2} \operatorname{Tr}\left(M^{\prime} M^{\prime \dagger}\right) \operatorname{Tr}\left(M M^{\prime \dagger}\right)+d_{3}\left(\operatorname{det} M^{\prime}+\text { h.c. }\right)+d_{4}^{a} \operatorname{Tr}\left(M^{\prime} M^{\prime \dagger} M^{\prime} M^{\prime \dagger}\right)+d_{4}^{b}\left(\operatorname{Tr}\left(M^{\prime} M^{\prime \dagger}\right)\right)^{2} \\
& +e_{2}\left(\operatorname{Tr}\left(M M^{\prime \dagger}\right)+\text { h.c. }\right) \\
& +e_{3}^{a}\left(\epsilon_{a b c} \epsilon^{\text {def }} M_{d}^{a} M_{e}^{b} M_{f}^{\prime c}+\text { h.c. }\right)+e_{3}^{b}\left(\epsilon_{a b c} \epsilon^{\text {def }} M_{d}^{a} M_{e}^{\prime b} M_{f}^{\prime c}+\text { h.c. }\right)
\end{aligned}
$$




$$
\begin{aligned}
& +e_{4}^{a} \operatorname{Tr}\left(M M^{\dagger} M^{\prime} M^{\prime \dagger}\right)+e_{4}^{b} \operatorname{Tr}\left(M M^{\prime \dagger} M^{\prime} M^{\dagger}\right) \\
& +e_{4}^{c}\left[\operatorname{Tr}\left(M M^{\prime \dagger} M M^{\prime \dagger}\right)+\text { h.c. }\right]+e_{4}^{d}\left[\operatorname{Tr}\left(M M^{\dagger} M M^{\prime \dagger}\right)+\text { h.c. }\right]+e_{4}^{e}\left[\operatorname{Tr}\left(M^{\prime} M^{\prime \dagger} M^{\prime} M^{\dagger}\right)+\text { h.c. }\right] \\
& +e_{4}^{f} \operatorname{Tr}\left(M M^{\dagger}\right) \operatorname{Tr}\left(M^{\prime} M^{\prime \dagger}\right)+e_{4}^{g} \operatorname{Tr}\left(M M^{\prime \dagger}\right) \operatorname{Tr}\left(M^{\prime} M^{\dagger}\right)+e_{4}^{h}\left[\left(\operatorname{Tr}\left(M^{\prime} M^{\prime \dagger}\right)\right)^{2}+\text { h.c. }\right] \\
& +e_{4}^{i}\left[\operatorname{Tr}\left(M M^{\dagger}\right) \operatorname{Tr}\left(M M^{\prime \dagger}\right)+\text { h.c. }\right]+e_{4}^{j}\left[\operatorname{Tr}\left(M^{\prime} M^{\prime \dagger}\right) \operatorname{Tr}\left(M^{\prime} M^{\dagger}\right)+\text { h.c. }\right] .
\end{aligned}
$$

Notice that among these terms, those with the coefficients $c_{2}, c_{4}^{a}, c_{4}^{b}, d_{4}^{a}, d_{4}^{b}, e_{3}^{a}, e_{4}^{a}, e_{4}^{b}, e_{4}^{f}, e_{4}^{g}$ and $e_{4}^{h}$ are $U(1)_{A}$ invariant. It also may be of some interest to write down the twenty one renormalizable terms, linear in the matrix $\mathrm{A}$, which transform like the QCD quark mass terms under $\mathrm{SU}(3) \times \mathrm{SU}(3)$. Again, for this listing, the $\mathrm{U}(1)_{A}$ transformation property of the mass terms in the fundamental QCD Lagrangian is respected only for the terms with the coefficients $k_{1}, k_{3}, k_{4}, k_{9}, k_{11}, k_{12}, k_{17}, k_{21}$.

$$
\begin{aligned}
V_{S B}= & +k_{1}[\operatorname{Tr}(A M)+\text { h.c. }]+k_{2}\left[\operatorname{Tr}\left(A M^{\prime}\right)+\text { h.c. }\right] \\
& +k_{3}\left[\operatorname{Tr}\left(A M M^{\dagger} M\right)+\text { h.c. }\right]+k_{4}\left[\operatorname{Tr}\left(A M M^{\prime \dagger} M^{\prime}\right)+\text { h.c. }\right] \\
& +k_{5}\left[\operatorname{Tr}\left(A M M^{\dagger} M^{\prime}\right)+\text { h.c. }\right]+k_{6}\left[\operatorname{Tr}\left(A M M^{\prime \dagger} M\right)+\text { h.c. }\right] \\
& +k_{7}\left[\operatorname{Tr}\left(A M^{\prime} M^{\prime \dagger} M^{\prime}\right)+\text { h.c. }\right]+k_{8}\left[\operatorname{Tr}\left(A M^{\prime} M^{\dagger} M\right)+\text { h.c. }\right] \\
& +k_{9}\left[\operatorname{Tr}\left(A M^{\prime} M^{\prime \dagger} M\right)+\text { h.c. }\right]+k_{10}\left[\operatorname{Tr}\left(A M^{\prime} M^{\dagger} M^{\prime}\right)+\text { h.c. }\right] \\
& +k_{11}[\operatorname{Tr}(A M)+\text { h.c. }] \operatorname{Tr}\left(M M^{\dagger}\right) \\
& +k_{12}[\operatorname{Tr}(A M)+\text { h.c. }] \operatorname{Tr}\left(M^{\prime} M^{\prime \dagger}\right) \\
& +k_{13}\left[\operatorname{Tr}(A M) \operatorname{Tr}\left(M M^{\prime \dagger}\right)+\text { h.c. }\right]+k_{14}\left[\operatorname{Tr}(A M) \operatorname{Tr}\left(M^{\prime} M^{\dagger}\right)+\text { h.c. }\right] \\
& +k_{15}\left[\operatorname{Tr}\left(A M^{\prime}\right)+\text { h.c. }\right] \operatorname{Tr}\left(M M^{\dagger}\right) \\
& +k_{16}\left[\operatorname{Tr}\left(A M^{\prime}\right)+\text { h.c. }\right] \operatorname{Tr}\left(M^{\prime} M^{\prime \dagger}\right) \\
& +k_{17}\left[\operatorname{Tr}\left(A M^{\prime}\right) \operatorname{Tr}\left(M M^{\prime \dagger}\right)+\text { h.c. }\right]+k_{18}\left[\operatorname{Tr}\left(A M^{\prime}\right) \operatorname{Tr}\left(M^{\prime} M^{\dagger}\right)+\text { h.c. }\right] \\
& +k_{19} A_{a}^{b} \epsilon_{b c d} \epsilon^{a e f} M_{e}^{c} M_{f}^{d}+\text { h.c. } \\
& +k_{20} A_{a}^{b} \epsilon_{b c d} \epsilon^{a e f} M_{e}^{\prime c} M_{f}^{\prime d}+\text { h.c. } \\
& +k_{21} A_{a}^{b} \epsilon_{b c d} \epsilon^{a e f} M_{e}^{c} M_{f}^{\prime d}+\text { h.c. }
\end{aligned}
$$

\section{APPENDIX B: SOME ISOSPIN RELATIONS}

We give examples of relations which follow from isotopic spin invariance:

$$
\begin{aligned}
\left\langle\frac{\partial^{2} V}{\partial \phi_{2}^{2} \partial \phi_{2}^{2}}\right\rangle & =\left\langle\frac{\partial^{2} V}{\partial \phi_{1}^{1} \partial \phi_{1}^{1}}\right\rangle, \\
\left\langle\frac{\partial^{2} V}{\partial \phi_{2}^{2} \partial \phi_{3}^{3}}\right\rangle & =\left\langle\frac{\partial^{2} V}{\partial \phi_{1}^{1} \partial \phi_{3}^{3}}\right\rangle, \\
\left\langle\frac{\partial^{2} V}{\partial \phi_{1}^{2} \partial \phi_{2}^{1}}\right\rangle & =\left\langle\frac{\partial^{2} V}{\partial \phi_{1}^{1} \partial \phi_{1}^{1}}\right\rangle-\left\langle\frac{\partial^{2} V}{\partial \phi_{1}^{1} \partial \phi_{2}^{2}}\right\rangle .
\end{aligned}
$$

Similar relations hold when $\mathrm{V}$ is differentiated with respect to two primed fields and with respect to one primed and one unprimed field.

[1] See the dedicated conference proceedings, S. Ishida et al "Possible existence of the sigma meson and its implication to hadron physics", KEK Proceedings 2000-4, Soryyushiron Kenkyu 102, No. 5, 2001. Additional points of view are expressed in the proceedings, D. Amelin and A.M. Zaitsev "Hadron Spectroscopy", Ninth International Conference on Hadron Spectroscopy, Protvino, Russia(2001) and A. H. Fariborz, "Scalar mesons, an interesting puzzle for QCD", Utica N. Y. (2003), AIP Conference Proceedings Vol. 688.

[2] E. van Beveren, T.A. Rijken, K. Metzger, C. Dullemond, G. Rupp and J.E. Ribeiro, Z. Phys. C 30, 615 (1986). E. van Beveren and G. Rupp, hep-ph/9806246 248. See also J.J. de Swart, P.M.M. Maessen and T.A. Rijken, U.S./Japan Seminar on the YN Interaction, Maui, 1993 [Nijmegen report THEF-NYM 9403].

[3] D. Morgan and M. Pennington, Phys. Rev. D 48, 1185 (1993). 
[4] A.A. Bolokhov, A.N. Manashov, M.V. Polyakov and V.V. Vereshagin, Phys. Rev. D 48, 3090 (1993). See also V.A. Andrianov and A.N. Manashov, Mod. Phys. Lett. A 8, 2199 (1993). Extension of this string-like approach to the $\pi K$ case has been made in V.V. Vereshagin, Phys. Rev. D 55, 5349 (1997) and in A.V. Vereshagin and V.V. Vereshagin, ibid. 59, $016002(1999)$.

[5] N.N. Achasov and G.N. Shestakov, Phys. Rev. D 49, 5779 (1994).

[6] R. Kamínski, L. Leśniak and J. P. Maillet, Phys. Rev. D 50, 3145 (1994).

[7] F. Sannino and J. Schechter, Phys. Rev. D 52, 96 (1995).

[8] N.A. Törnqvist, Z. Phys. C 68, 647 (1995) and references therein. In addition see N.A. Törnqvist and M. Roos, Phys. Rev. Lett. 76, 1575 (1996), N.A. Törnqvist, hep-ph/9711483 and Phys. Lett. B 426105 (1998).

[9] R. Delbourgo and M.D. Scadron, Mod. Phys. Lett. A 10, 251 (1995). See also D. Atkinson, M. Harada and A.I. Sanda, Phys. Rev. D 46, 3884 (1992).

[10] G. Janssen, B.C. Pearce, K. Holinde and J. Speth, Phys. Rev. D 52, 2690 (1995).

[11] M. Svec, Phys. Rev. D 53, 2343 (1996).

[12] S. Ishida, M.Y. Ishida, H. Takahashi, T. Ishida, K. Takamatsu and T Tsuru, Prog. Theor. Phys. 95, 745 (1996), S. Ishida, M. Ishida, T. Ishida, K. Takamatsu and T. Tsuru, Prog. Theor. Phys. 98, 621 (1997). See also M. Ishida and S. Ishida, Talk given at 7th International Conference on Hadron Spectroscopy (Hadron 97), Upton, NY, 25-30 Aug. 1997, hep-ph/9712231

[13] M. Harada, F. Sannino and J. Schechter, Phys. Rev. D 54, 1991 (1996).

[14] M. Harada, F. Sannino and J. Schechter, Phys. Rev. Lett. 78, 1603 (1997).

[15] D. Black, A.H. Fariborz, F. Sannino and J. Schechter, Phys. Rev. D 58, 054012 (1998).

[16] D. Black, A.H. Fariborz, F. Sannino and J. Schechter, Phys. Rev. D 59, 074026 (1999).

[17] J.A. Oller, E. Oset and J.R. Pelaez, Phys. Rev. Lett. 80, 3452 (1998). See also K. Igi and K. Hikasa, Phys. Rev. D 59, 034005 (1999).

[18] A.V. Anisovich and A.V. Sarantsev, Phys. Lett. B 413, 137 (1997).

[19] V. Elias, A.H. Fariborz, Fang Shi and T.G. Steele, Nucl. Phys. A 633, 279 (1998).

[20] V. Dmitrasinović, Phys. Rev. C 53, 1383 (1996).

[21] P. Minkowski and W. Ochs, Eur. Phys. J. C 9, 283 (1999).

[22] S. Godfrey and J. Napolitano, hep-ph/9811410

[23] L. Burakovsky and T. Goldman, Phys. Rev. D 572879 (1998)

[24] A. H. Fariborz and J. Schechter, Phys. Rev D 60, 034002 (1999).

[25] T. Hatsuda, T. Kunihiro and H. Shimizu, Phys. Rev. Lett. 82, 2840 (1999); S. Chiku and T. Hatsuda, Phys. Rev. D 58, 076001 (1998).

[26] D. Black, A. H. Fariborz and J. Schechter, Phys. Rev. D 61074030 (2000). See also V. Bernard, N. Kaiser and U-G. Meissner, ibid 443698 (1991).

[27] D. Black, A. H. Fariborz and J. Schechter, Phys. Rev. D 61074001 (2000).

[28] L. Celenza, S-f Gao, B. Huang and C.M. Shakin, Phys. Rev. C 61, 035201 (2000).

[29] D. Black, A.H. Fariborz, S. Moussa, S. Nasri and J. Schechter, Phys.Rev. D 64, 014031 (2001).

[30] M. Harada, F. Sannino and J. Schechter, Phys. Rev. D 69, 034005 (2004). See also J. R. Pelaez, Phys. Rev. Lett. 92, 102001 (2004) hep-ph/0309292 ; and M. Uehara, arXiv:hep-ph/0308241

[31] R. L. Jaffe, Phys. Rev. D 15, 267 (1977).

[32] In addition to 27] and [29] above see T. Teshima, I. Kitamura and N. Morisita, J. Phys. G. 28, L391 (2002); F. Close and N. Tornqvist, ibid. 28, R249 (2002) and A. H. Fariborz, Int. J. Mod. Phys. A 19, 2095 (2004); 19, 5417 (2004).

[33] M. Gell-Mann and M. Lévy, Nuovo Cimento 16, 705 (1960).

[34] J. Cronin, Phys. Rev. 161, 1483 (1967); S. Weinberg, Phys. Rev. Lett 18, 188 (1967).

[35] J. Gasser and H. Leutwyler, Ann. Phy. (NY) 158, 142, (1984); Nucl. Phy. B 250, 465, (1985). A more recent review is given by U-G. Meissner, Rep. Prog. Phys. 56, 903 (1993).

[36] S. Weinberg, Phys. Rev. Lett. 17, 616 (1966).

[37] M. Lévy, Nuovo Cimento A 52, 23 (1967). See S. Gasiorowicz and D. A. Geffen, Rev. Mod. Phys. 41, 531 (1969) for a review which contains a large bibliography.

[38] J. Schechter and Y. Ueda, Phys. Rev. D 3, 2874, 1971; Erratum D 8987 (1973). See also J. Schechter and Y. Ueda, Phys. Rev. D 3, 168, (1971).

[39] G.'t Hooft, Phys. Rev. Lett. 37, 8 (1976).

[40] V. Mirelli and J. Schechter, Phys. Rev. D 15, 1361 (1977).

[41] G.'t Hooft, arXiv:hep-th/9903189

[42] M. Napsuciale and S. Rodriguez, Phys. Rev. D 70, 094043 (2004), arXiv:hep-ph/0407037

[43] N. Isgur and J. Weinstein, Phys. Rev. Lett 48, 659 (1982); Phys. Rev. D 27588 (1983). See also F.E. Close, N. Isgur and S. Kumano, Nucl. Phys. B 389, 513 (1993).

[44] C. Rosenzweig, J. Schechter and G. Trahern, Phys. Rev. D 21, 3388 (1980); J. Schechter, Phys. Rev. D 21, 3393 (1980); P. Di Vecchia and G. Veneziano, Nucl. Phys. B 171, 253 (1980); P. Nath and R. Arnowitt, Phys. Rev. D 23, 1789 (1981); E. Witten, Ann. Phys. (N.Y.) 128, 363 (1980); A. Aurilia, Y. Takahashi and D. Townsend, Phys. Lett. B 95, 265 (1980); K. Kawarabayashi and N. Ohta, Nucl. Phys. B 175, 477 (1980); ibid Prog. Theor. Phys. 66, 1789 (1981).

[45] S. D. H. Hsu, F. Sannino and J. Schechter, Phys. Lett. B 427, 300 (1998).

[46] Isospin violation is included in the single chiral nonet version of this model in J. Schechter and Y. Ueda, Phys. Rev. D 4, 733 (1971). 
[47] Particle Data Group, Phys. Lett. B 5921 (2004).

[48] J. Schechter and H. Weigel, Phys. Rev. D 44, 2916 (1991); Phys. Lett. B 261, 235 (1991); H. Weigel, Eur. Phys. J. A 2, 391 (1998).

[49] R. Dashen, Phys. Rev. 183, 1245 (1969). 